\title{
Can we prevent the transmission of tuberculosis?
}

Current strategies to reduce the burden of tuberculosis (TB) are symptom- and patient-centred, and mainly focus on accurate diagnosis of cases, adequate follow-up, adherence to treatment and contact tracing.

This has shown modest success, with the global TB burden dropping at an average of $1.8 \%$ annually since $2001 .^{[1]}$ The goals of the World Health Organization (WHO)'s End TB strategy include reducing the incidence of TB disease by $50 \%$ by 2025 and $90 \%$ by 2035. ${ }^{[2]}$ To achieve this, the drop in the global burden of TB disease needs to improve to a $10 \%$ annual reduction. Clearly, improved tools and interventions are required urgently.

An article in the current issue of SARJ, by Calligaro et al., ${ }^{[3]}$ 'The diagnostic gap: Characterising the profile of undiagnosed infectious TB patients residing in the community', highlights the importance of innovative new approaches to reduce TB incidence, and identifies a possible target population for such new interventions. They cite references pointing to important issues including the following: (i) roughly $30-50 \%$ of the $\mathrm{TB}$ disease burden is undiagnosed; (ii) transmission often occurs outside the home, in places where the community congregates during daily living. They performed active case finding in some of these suspected high-transmissionrisk public areas, and found a higher than anticipated prevalence of active disease. In addition, they found that almost half of these cases were acid-fast bacilli smear-positive, indicating a highly infectious potential. Furthermore, all these cases were symptomatic. It is not clear why these community members failed to seek healthcare, in spite of living in an area where facilities are relatively accessible.

In HIV-positive patients, intensified screening, prophylactic treatment of TB and campaigns to reduce the prevalence of HIV have yielded success, yet no other high-risk groups have been targeted. Other possible high-risk groups include people with known exposure to active cases, people frequenting transmission hotspots, people with previous TB episodes, smokers, diabetics and alcoholics.
Proactive campaigns are crucial to allow earlier detection and treatment, which will greatly reduce the transmissions originating from a single case. Urgent research and pilot programmes to test the effectiveness and cost-effectiveness of systematic screening interventions are required. The shortcomings of available testing options, however, play a major role in the lack of such interventions. Screening programs would be made more feasible by improved tools, such as a point-of-care test that does not require sputum. In addition, it could provide the added benefit of detecting TB cases prior to infectious risk and lung damage.

To reach the ambitious targets of the WHO End TB strategy, it is clear that out-of-the-box solutions will have to be explored. The work by Calligaro et al. ${ }^{[3]}$ suggests that one such approach might be the interruption of transmission by screening and initiation of treatment in people who have not yet self-reported to primary health care services, in high TB prevalence communities.

\section{Stephanus T Malherbe \\ Gerhard Walzl}

Department of Science and Technology/National Research Foundation Centre of Excellence for Biomedical Tuberculosis Research and South African Medical Research Council Centre for Tuberculosis Research and Division of Molecular Biology and Human Genetics, Faculty of Medicine and Health Sciences, Stellenbosch University, Cape Town, South Africa

\section{malherbe@sun.ac.za}

1. World Health Organization. Global Tuberculosis Report. 2015. http://www.who.int/ tb/publications/global_report/en/ (28 October 2016)

2. World Health Organization. The End TB Strategy. 2015. http://www.who.int/tb/ strategy/en (28 October 2016)

3. Calligaro G, Esmail A, Mnguni T, Mottay L, Dheda K. The diagnostic gap: Characterising the profile of undiagnosed infectious TB patients residing in the community. S Afr Resp J 2016;22(4):93-98. http://dx.doi.org/10.7196/SARJ.2016.v22i4.100

S Afr Respir J 2016;22(4):91. DOI:10.7196/SARJ.2016.v22i4.106 